\title{
TOXIC EFFECTS OF CHLOROPICRIN AND IMPACT OF SORBED WATER STEAM ON PROTECTION
}

\author{
Milena Nikolić, Faculty of Medical Sciences, Kragujevac, Serbia milena_nikolic@yahoo.com \\ Mladen Nikolić, College for chemical-technological school, Kruševac, Serbia mladennikolic2603@yahoo.com \\ Dragan Nikolić, College for chemical-technological school, Kruševac, Serbia dragannikolic60@ yahoo.com
}

\begin{abstract}
Chloropicrin is a chemical substance that has a very toxic effect. Exerts its effect on the respiratory system. Causes pulmonary edema and difficult breathing and suffocating effect. Respiratory protection may be carried into execution respiratory filters. On the protective power filter based on active coal affects adsorbed water vapor. This paper presents the results of the adsorption of water vapor on activated carbon from 5\% to $25 \%$. Was used for the experiment apparatus for dynamic adsorption, the results showed that the humidity of 5\% coal provides most power protection, while humidity of $25 \%$ minimum protective power.
\end{abstract}

Keywords: toxic, Chloropicrin, filter, adsorption, water vapor, relative humidity, activated carbon, protective power

\section{Toxic effects of Chloropicrin}

Chloropicrin was first obtained by the action of chlorine lime picric acid. Besides insecticide Chloropicrin effect acts as a fungicide, herbicide and nematocid. Has an important application in soil disinfection.

Restricted used for disinfection and storage space due to its toxicity to humans.

Chloropicrin is a colorless oily liquid and a characteristic pungent odor. Obtained by light green-yellow color due to decomposition of chlorine and nitrogen oxides. Solubility in water is $0.16 \%$. In organic solvents to dissolve well.

Chloropicrin is stable compound and a derivative of methane preserved the properties of saturated hydrocarbons.At elevated temperature and the boiling temperature of slightly decomposed. Chloropicrin hydrolysis does not take place in an aqueous medium, even at elevated temperatures.

Chloropicrin is a strong poison that adversely affects the lungs. Liquid Chloropicrin causes severe damage to the skin. Concentration of $0.002 \mathrm{mg} / \mathrm{dm} 3$ suzavačka cause of action for a period of 3 to
30 seconds, and the concentration of 0.05 $\mathrm{mg} / \mathrm{dm} 3$ was unbearable. Higher concentrations cause nausea, vomiting and stomach.Damage to the respiratory system occurs at concentrations greater than 0.1 $\mathrm{mg} / \mathrm{dm} 3$, and death at a concentration of 2 $\mathrm{mg} / \mathrm{dm} 3$ and exposure of 10 minutes.

The main mechanism of action is occurrence of pulmonary edema. Pulmonary edema occurs due to disturbance alveolar capillary permeability and causing the output of fluid from the blood into the alveolar spaces. The increase in lung vascular permeability is the result of direct action of the poison and disorder nervous regulation of capillary function. Poison, and the reaction products act on the receptors of the lungs, where the vagus nerve impulse is sent through the central nervous system. Effector impulses back to the effector organ via the sympathetic nerves. Reflex defenses are manifested by creating fluid in the alveoli lungs. In further reflection of the pathologic character, which leads to an increase in pulmonary edema. The occurrence of pulmonary edema initially difficult, and later disables reception of oxygen from the blood into the alveoli and excretion of carbonic acid from the blood into the alveoli. Hemoglobin oxygen saturation decreases and at the moment of death is almost no oxygen.

\section{Protection from Chloropicrin}

Respiratory protection of Chloropicrin may be provided with respirators. Filling most suitable respirator is activated carbon.

Activated carbon is one of a group of materials that have a highly developed porosity. Carbon atoms are held together by covalent bonds, are arranged in a plane in the 
form of hexagonal rings. The distance between the planes is higher than that of graphite. A number of these two planes placed one above the other, form crystallites. Crystallites of activated carbon are each different in size, they are not properly lined up next to each other, and therefore have high surface heterogeneity.

Micropores are the smallest pores with a diameter below 15-16 x 10-10 m. Based on tests Dubinina, a large number of microporous activated carbons have micropore diameters in the range of $4-8 x$ 10-10 $\mathrm{m}$. The size of these pores coincides with the size of molecules adsorbujućih.

Micropore volume of activated carbons ranges from 0.20 to $0.60 \mathrm{~cm} 3 / \mathrm{g}$, a surface area of $1000-2000 \mathrm{~m} 2$ per gram.

Transitional pores larger than micropores and have diameters of 15 - 16 to 1000 to $2000 \times 10-10 \mathrm{~m}$.

Transition pore volume of activated carbons was in the range of 0.02 to $0.10 \mathrm{~cm} 3$ / g. Area per gram in the range of $20-70 \mathrm{~m} 2$. Effective transition pore diameters are in the range of 40-1000 x 10-10 m.

Have a radius of macropores above 1000 - $2000 \times 10-10$ we are most massive pores of activated carbon. Macropore volume ranges from 0.2 to $0.8 \mathrm{~cm} 3 / \mathrm{g}$, and the area per gram within the limits of $0.5-2 \mathrm{~m} 2$.

Some carbon atoms in activated carbon are not at the same energy level. These include unsaturated carbon atoms valence that allows chemical bonding with other molecules, atomic groups and radicals.

Gaseous substances adsorbed onto activated carbon is of particular importance to oxygen. Oxygen can be adsorbed onto activated carbon in two basic ways:

- physically adsorbed and

- chemisorbed.

Which of these modes dominates, depends on temperature. At lower temperatures, the oxygen is adsorbed mainly physical adsorption, and as the temperature increases, and increases the reaction hemisorpcije, where oxygen molecules are dissociated into atoms, which react chemically with the atoms of coal and generate the surface oxide layer.
Oxidized surface, formed at hemisorpciji oxygen at high temperatures, unless there is a shift in the gas phase, they differ from those of stability of chemisorption formed at lower temperatures. In this case, there is a great diversity of surface oxygen composition. When the surface of activated carbon oxidized atmospheric oxygen, at a temperature of about 300 OC hemisorpcije process there is an increase, and the maximum level is formed at a temperature of 400-450 0C. At higher temperatures, the oxide breaks down into several parts, and at a temperature of 900 - 1000 0C most of the unbuilt.

Carbon surface can be reduced by hydrogen at high temperature, or increasing hydrogen pressure.

Presence of chemisorbed oxygen on the surface of carbonaceous substances has an important effect on the adsorption of water vapor.

Pierce and Smith described the adsorption of water, where the water molecules bind to the primary adsorption centers. The adsorbed water molecules are subsequently act as secondary centers, which builds multimolekularna the adsorption pressure increased.

When the pressure is sufficiently high, the micropores are filled, and with further increase in pressure multimolekularna adsorption continues to create the surface of the membrane, and in large enough pore capillary condensation builds, the water vapor pressure sufficient for saturation.

Dubinin and coworkers have found that the work for the process of interaction of active carbon and oxygen characteristic two extreme cases:

- At a temperature of 0 - 1000C on activated carbon surface oxides are formed the base character and

- At temperatures above 2000C the interaction of activated carbon and oxygen leads to the formation of surface oxides primarily acidic character.

Some research has shown that the presence of acidic surface oxides of abnormal adsorption capacity of activated carbons by polar molecules. 
Dubinin and conspiracy investigated the adsorption isotherm of water on samples of activated carbon. They found that there is a substantial influence of surface oxides on the ability of activated carbon with absorption by water vapor.

Dubinin and coworkers laid the basis of the theory of adsorption of water vapor, based on hemisorpciji the primary adsorption centers, ie, the surface oxides.

Water chemisorbed on oxides, via hydrogen bonds, creating new secondary centers suitable for further chemisorption.

Both types of surface oxides, especially acidic character, show a significant influence on the adsorption of water vapor on activated carbon. At relative humidity greater than 40-50\%, water vapor molecules bind with the free polar centers of the lattice and the previously adsorbed water molecules through hydrogen bridges, so that a significant increase in the adsorption of water vapor, which can be seen on the adsorption isotherms of water vapor.

When the carbon surface is covered with almost complete monomolecular covers, with a further increase of the relative pressure, are formed Pore volume is filled at the point when the water vapor is adsorbed on opposite walls combination. When activated charcoal that does not contain a proportional relationship transition pore volume of adsorbed water vapor at saturation pressure is equal to the volume of micropores. Hysteresis loop can not be compared with the capillary condensation of water vapor, because the same thing happens on activated carbon, which is no transition pore, and hysteresis loops show a striking effect of molecular sieves.

When activated carbons that have developed transitional pores, they become full-induced capillary condensation at high relative pressures, it becomes a permanent case of monomolecular and multimolekularne adsorption on the walls of the pores.

Water vapor adsorption on activated carbon with developed porosity transition progresses in two phases hysteresis on adsorption isotherms. At lower relative pressures, a hysteresis loop is determined by the polarity of groups at higher relative pressures, capillary condensation in transitional pores.

Kiselov and his colleagues concluded that the adsorption of water vapor on activated carbon, depending on the degree of oxidation of the surface and porosity of coal, and that the primary role in the adsorption of water vapor capillary condensation.

Daceya, Clunier and Thomas during the experiment, water vapor adsorption on activated carbon, found no hysteresis loop which explains the high microporous structure of activated carbon.

Research adsorption of water vapor state that is the same as a function of chemical structure and porosity of activated carbon and also quite far from the end of the possible solutions. However adsorbed water vapor, and to a certain extent and relative humidity affect the protective power, or on the adsorption of toxic substances within the meaning of increase or decrease in the protective power which depends on the physico-chemical properties of adsorbing matter.

\section{Experimental research}

Used for the experiments of the apparatus shown in Figure 1 The apparatus was composed of the following parts: 1 Rotometar air, 2 Court with concentrated sulfuric acid, 3 Court with water, 4.Psihrometar, 5.Mixer , 6 Rotometar for secondary air, 7 Wash with concentrated sulfuric acid, with a dry 8.T ower solid adsorbent, 9 Wash with Chloropicrin, 10 Way stop cock, 11 Respiratory filter, 12 Respiratory filter to be tested, 13.Collon with dry activated carbon, 14 Quartz heating tube, 15 indicator 


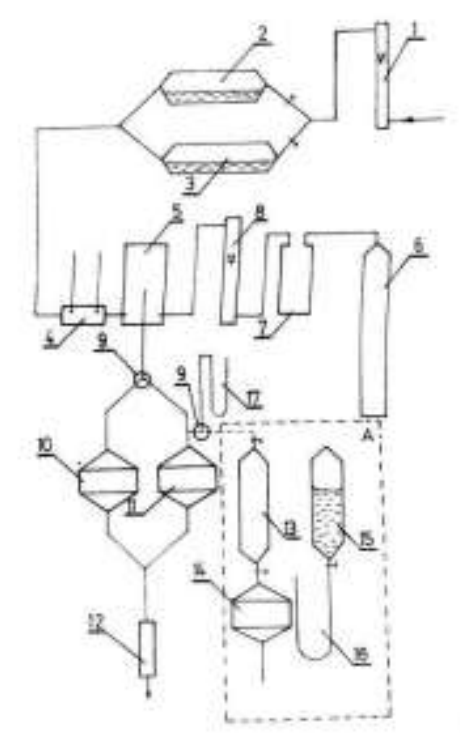

Figure 1. Aparatus for filter testing

Through the respiratory filter is a mixture of vapor permeable Chloropicrin and air mixture at a flow rate of $0.5 \mathrm{dm} 3 / \mathrm{s}$ with a concentration of $0.5 \%$ Chloropicrin Cmdr. The amount of air mixed with $24 \mathrm{dm} 3 / \mathrm{min}$ air mixer and passed through respiratory filter. Chloropicrin mixture of air and breaking through the respiratory filter is carried out through a quartz tube that is directly heated burner, and then sent to indicator. Chloropicrin when going through the quartz tube leads to its destruction. Chlorine, which is created by decomposition of Chloropicrin in contact with the indicator as active iodine from potassium iodide suppressed and newly formed elemental iodine with starch builds a blue discoloration of the end of the experiment.

\section{Results and discussion}

The effects of water vapor adsorption on activated carbon in the respiratory protective power filter are given in Table 1 and Graph 1.

From the table (1) and graphics (1) shows that the adsorbed water vapor on activated carbon has an impact on the protective power of Chloropicrin filter. Increasing water vapor adsorption on activated carbon adversely affects the protective power filters. Adsorbed water vapor adsorption space is occupied by active carbon, so that the cut surface of activated carbon, which is available Chloropicrin. Chloropicrin is not susceptible to hydrolysis, which further reduces the adsorption power of coal. In Respiratory protection of Chloropicrin should strive to charcoal filters, which are filled with a minimum sorbed water vapor.

Table (1) Effect of water vapor adsorbed at a relative humidity of $50 \%$ in the protective power

\begin{tabular}{cccc}
\hline $\begin{array}{c}\text { Moisture of } \\
\text { activated } \\
\text { charcoal } \\
(\%)\end{array}$ & $\begin{array}{c}\text { Relative } \\
\text { humidity } \\
(\%)\end{array}$ & \multicolumn{2}{c}{$\begin{array}{c}\text { The protective } \\
\text { power of filter } \\
\text { min }\end{array}$} \\
\hline 5 & 50 & 48 & 32 \\
\hline 10 & 50 & 45 & 15 \\
\hline 15 & 50 & 43 & 40 \\
\hline 20 & 50 & 39 & 35 \\
\hline 25 & 50 & 37 & 27 \\
\hline
\end{tabular}




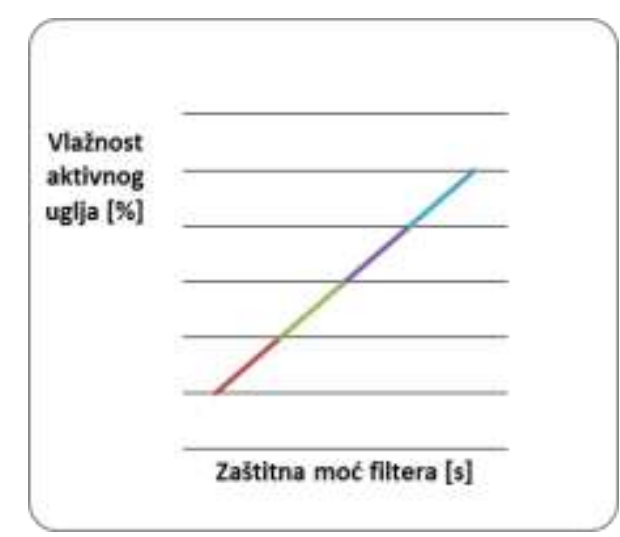

Graph 1. Protective power of time to Chloropicrin filters depending on the adsorption of water vapor

\section{Conclusion}

For filtration of air contaminated with Chloropicrin can be used filters based on activated carbon. Aivated charcoal may have, depending on usage conditions, different humidity, and can be used at different relative humidity. Desorbed water vapor on activated carbon was important for the sorption of Chloropicrin. chloropicrin is not susceptible to hydrolysis and adsorbed water vapor adversely affects the protective power filters. The study showed that activated charcoal humidity of $5 \%$ provides protective power of $48 \mathrm{~min}$ and $32 \mathrm{~s}$, while activated charcoal humidity of $25 \%$ reduces the protective power of filters at $37 \mathrm{~min}$ and $27 \mathrm{~s}$.

\section{References}

1.Brajović M.: Chemistry of poison gases, Belgrade (1983)

2.D. Nikolic, R. Biočanin: activated carbon NBC protection function, Bulletin NBC, Krusevac (1995)

3.D. Nikolic: Influence of relative vlažosti air purification air pollution in order to protect the ecosystem, Ekologika 3, (1996)

4.D. Nikolic: Protection against the effects of toxic substances and effects of NBC weapons, SC NBC Krusevac (1997)

5.Nikolic D.: Geometry of filters for respiratory protection, environment and ecosystem from toxic gases and vapors, Bulletin NBC, Krusevac (1996)

6.Stanković D.: Medicine, Medical Books Belgrade-Zagreb (1986) 